\title{
Topical lidocaine $2 \%$ gel for analgesia and patient comfort during interscalene brachial plexus and axillary blocks: $A$ noninferiority randomized controlled trial
}

Cédric Bouts, Sam Van Boxstael, Catherine Vandepitte, Alexander Vloka, Ine Leunen, Hassanin Jalil, Max Kuroda

Department of Anesthesiology, Intensive Care, Emergency Medicine and Pain Therapy, Ziekenhuis Oost-Limburg Genk, Belgium

\section{Background and goal of study}

The interscalene (ISBP) and axillary (AX) brachial plexus blocks are common anesthetic methods for upper limb surgery. Needle insertion and manipulation during the blocks may cause substantial discomfort that can increase anxiety, fear and dissatisfaction with anesthesia care. ${ }^{1}$ Topically applied local anesthetic gel could provide analgesia while eliminating the need for additional injections. ${ }^{2,3}$ The primary objective of this study was a noninferiority comparison between the analgesia achieved with topical gel to that of skin infiltration for pain upon needle insertion and injection during administration of the ISBP and AX brachial plexus blocks.

\section{Materials and methods}

After EC approval (B371201628396), 30 patients undergoing an ISBP or AX block for surgery of the upper limb were randomized into one of three groups: no anesthesia prior to nerve block, skin infiltration with $3 \mathrm{~mL}$ lidocaine $2 \% 5 \mathrm{~min}$ prior to nerve block, or $10 \mathrm{~mL}$ topical lidocaine $2 \%$ gel applied to the skin surface over the interscalene space $5 \mathrm{~min}$ prior to nerve block. Primary outcome variables were Visual Analogue Scale (VAS) pain scores during needle insertion and injection. To help clarify the role of patient discomfort on pain ratings, fearfulness on a 5point Likert scale (from 1 no fear to 5 very fearful) and global discomfort assessed as Verbal Rating Scale (VRS) scores (from 0 more comfortable / less painful than expected to 10 less comfortable / more painful than expected) were also evaluated.

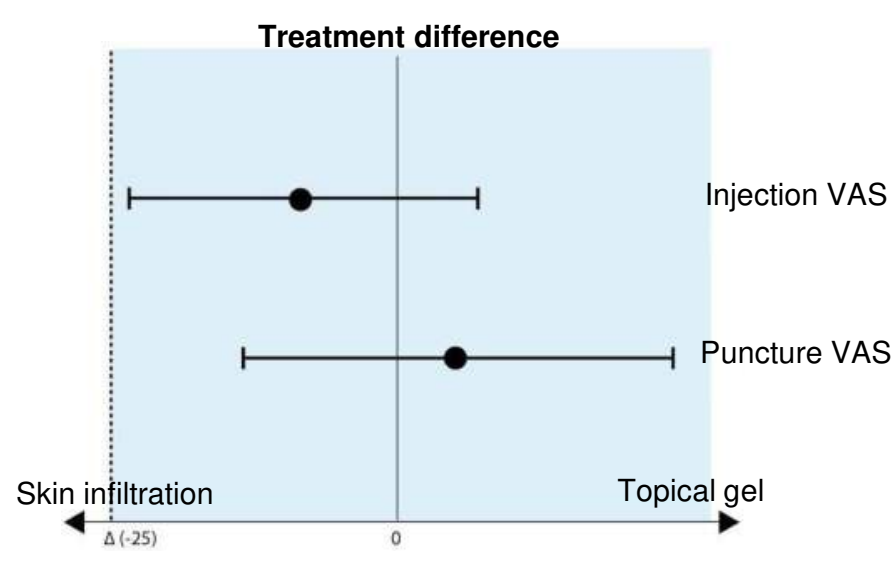

\section{Results and Discussion}

Topical lidocaine $2 \%$ gel was found to be noninferior to lidocaine infiltration in improving pain during needle insertion and injection of the ISBP and AX blocks. Fearfulness significantly increased needle insertion VAS $(5.6 \pm 2.0,95 \% \mathrm{Cl} 1.5-9.7$, adjusted $p=$ $0.01)$.

\section{Conclusion}

Analgesia conferred by application of lidocaine gel was noninferior to that of infiltration with lidocaine $2 \%$ for pain during needle insertion and injection of the ISBP and AX blocks. Lidocaine gel does not require the extra needle insertion and injection that can add to patient discomfort and can increase the risk for intraneural or intravascular injection. Moreover, when applied topically, gel containing local anesthetic may be timesaving as the gel can also serve as an ultrasound acoustic coupling medium. Thus sterile lidocaine $2 \%$ gel may confer adequate analgesia and ultrasound images for the ISBP and AX block procedures, while eliminating the need for additional injections and time required to apply more sterile gel.

Table. Pain on puncturs, pain on injection and global patient comfort during 1 SB and $A$ xe

\begin{tabular}{|c|c|c|c|c|}
\hline & $\begin{array}{l}\text { Control } \\
\{n=10)\end{array}$ & $\begin{array}{c}\text { Lidondane infitration } \\
(n=10)\end{array}$ & $\begin{array}{c}\text { Listoratite get } \\
(n=10)\end{array}$ & 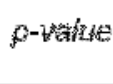 \\
\hline \multirow{4}{*}{ 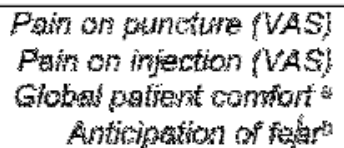 } & $17 \pm 14$ & $15 \pm 14$ & $23 \pm 1 \hat{3}$ & $n s$ \\
\hline & $25 \pm 21$ & $24 \pm 18$ & $19 \pm 15$ & 19S \\
\hline & $2 \pm 2$ & $2 \pm 1$ & $1 \pm 2$ & $\mathrm{n}$ \% \\
\hline & $9 \pm 1$ & $1 \pm 1$ & $2 \pm 1$ & 0.128 \\
\hline
\end{tabular}

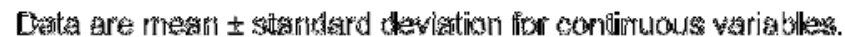

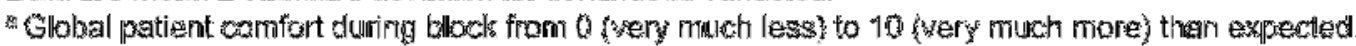

is Anticipation of fear from 1 (no fiear) to 5 (very fearful) 